\title{
A Relay Assisted Cooperative Transmission Protocol for Wireless Multiple Access Systems
}

\author{
Zhiguo Ding, Member, IEEE, Kin K. Leung, Fellow, IEEE, Dennis L. Goeckel, Senior Member, IEEE, and Don \\ Towsley, Fellow, IEEE
}

\begin{abstract}
In this paper, we propose a spectrally efficient cooperative transmission protocol for multiple access scenarios. The key feature is to utilize multi-user diversity and fully exploit the dynamic nature of radio propagation. In particular, by carefully scheduling the multiple sources and relays' transmissions, a source with a poor connection to the destination can have higher priority to obtain help from a relay with better channel condition. As a result, the full diversity gain is achievable even though only a fraction of relays is scheduled to help each user. We developed an achievable diversity-multiplexing tradeoff for the proposed transmission protocol to assist performance evaluation. When the number of relays is large, the diversity-multiplexing tradeoff achieved by the proposed scheme can approximate the optimal multiple-input single-output upper bound. Both analytical and numerical results show that the proposed protocol outperform other comparable schemes in most conditions.
\end{abstract}

\section{INTRODUCTION}

As an alternative to multiple-input multiple-output (MIMO) techniques, cooperative diversity offers an efficient and lowcost way to provide spatial diversity, which is effective in combating multipath fading in wireless environments [1]. By encouraging single-antenna nodes to cooperatively share their antennas, a virtual antenna array can be formed and the robustness of reception can be improved without requiring extra hardware cost. Initial studies focused on the basic scenario of a source-destination pair [2], [3]. More recent studies for cooperative diversity have focused on more realistic and practical communication scenarios, such as multiple access channels (MAC), broadcasting channels, interference channels and the two-way relaying scenario [4], [5]. For example, the use of relay selection has been applied to multi-source multi-destination scenarios in [6], which can achieve the full diversity gain but suffer some loss of multiplexing gains in order to avoid co-channel interference.

The multiple access channel is an important communication scenario and has been recognized as one of the fundamental building blocks for modern wireless networks [7], [8]. Its application can be found in wireless sensor networks, where

Research was sponsored by US Army Research laboratory and the UK Ministry of Defence and was accomplished under Agreement Number W911NF06-3-0001, and by the US National Science Foundation under grant CNS0721861. Z. Ding was supported by the UK EPSRC under grant number EP/F062079/1.

Zhiguo Ding is with Dept. of Communication Systems, Lancaster University, UK. Kin K. Leung is with Dept. of Electrical and Electronic Engineering, Imperial College, London, SW7 2BT, UK. Dennis L. Goeckel is with Dept. of Electrical and Computer Engineering, University of Massachusetts, Amherst, MA, US. Don Towsley is with Dept. of Computer Science, University of Massachusetts, Amherst, MA, US. a data fusion center collects data from multiple sensors, and in traditional cellular networks, where multiple users communicate with a base station. In [4] a cooperative multiple access (CMA) transmission protocol was proposed; similar work based on superimposed modulation can be found in [9]. In general, these CMA schemes only exploit cooperation among the source nodes and hence their achievable diversity gain is constrained by the number of source nodes. Recall that in a wireless network, the number of active users is typically much smaller than the idle ones, which can be utilized as dedicated relays. Hence it is of interest to invite idle nodes to cooperate as relays, and to exploit them as an extra dimension to further enhance the system robustness. However, relays are different from sources in the sense that they only repeat what has been transmitted, and hence it is desirable to reduce the bandwidth consumed by such relay transmission. This motivates the application of network coding over cooperative diversity to improve its spectral efficiency [10], [11]. However, for many network coded cooperative transmission schemes, the computational complexity at the intermediate relay nodes could be high due to the complicated operations for decoding and encoding the received mixture. Note that the idea of using dedicated relays has been previously proposed in [12], [13]. Specifically, provided that all nodes are only equipped with a single antenna, a study of the diversity-multiplexing tradeoff has been carried out for the multiple access scenario with one relay. And [12] the number of relays has to be assumed to be less than the number of source nodes.

In this paper, we propose a new relay-assisted cooperative transmission protocol for multiple access communication scenarios with dedicated relays. By carefully scheduling the transmissions of multiple sources and relays, a source with a poor connection to the destination is given a higher priority in order to obtain better service from the relays. Hence the maximum diversity gain becomes achievable for each user, with only a fraction of the relays scheduled to help any given user. A non-orthogonal transmission strategy is also applied, where the source transmission and relay forwarding take place at the same time. Since relay transmission is only to repeat what has been sent, it is desirable that the bandwidth resource consumed by relaying be as small as possible. By introducing non-orthogonal transmission, it becomes possible for relay forwarding and source transmission to share the same bandwidth, which ensures a more efficient use of bandwidth compared with orthogonal transmission schemes.

Diversity-multiplexing tradeoffs have been widely used to evaluate the spectral efficiency of MIMO techniques. Since a 
cooperative network can be viewed as a special case of MIMO, it is desirable to develop the diversity-multiplexing tradeoff for the purpose of performance evaluation. Following the definition of the MAC outage events provided in [8], the outage probability achieved by the proposed transmission protocol is studied. The fact that the sources and relays are no longer scheduled randomly, unlike the schemes in [2], [4], makes it difficult to obtain explicit expressions, since the channel coefficients are ordered in a certain way. Thus, order statistics [14] are applied here to obtain closed-form expressions of the outage probability. In particular, an achievable diversitymultiplexing tradeoff is developed for the proposed relayassisted CMA scheme. When the number of relays is large, we show that the diversity-multiplexing tradeoff achieved by the proposed scheme approaches the optimal tradeoff achieved by the classical multiple-input single-output scheme. Compared to the CMA schemes in [4], [9], the proposed scheme achieve a better diversity-multiplexing tradeoff in the low and intermediate multiplexing gain ranges, and eventually become the dominant scheme with a sufficiently large number of relays. Monte-Carlo simulation results also demonstrate the performance of the proposed transmission protocol.

This paper is organized as follows. The proposed cooperative multiple access transmission scheme is described in in Section II. The diversity-multiplexing tradeoff of the proposed protocol is studied in Section III and numerical results are provided. The details for the development of the diversitymultiplexing tradeoff are provided in Section IV. Finally, concluding remarks are given in Section V.

\section{DESCRIPTION OF THE COOPERATIVE MULTIPLE ACCESS CHANNEL SCHEME}

We consider a setting where $M$ source nodes deliver data to a common destination. In addition there are $L$ relay nodes helping the communication between the sources and destination. Each node is constrained by the half duplexing assumption, where one node can not transmit and receive at the same time. Time division multiplexing access is considered here due to its simplicity. All channels considered here are assumed to experience quasi-static identical independent Rayleigh fading. And all nodes, including the sources and relays, use the same transmission power.

\section{A. Initialization}

In this paper, the decode-forward (DF) strategy is used for relay transmission. We assume that each relay has the local channel state information (CSI) for the incoming and outgoing channels. Such CSI information can be obtained by asking the $M$ source nodes and the common destination to broadcast pilot signals. In total, $M+1$ time slots are required for such training signaling. Note that one time slot for training is unavoidable for the DF scenario with a single source-destination pair [2]. Given $M$ source nodes, $M$ time slots will be consumed also for the traditional scheme, and the proposed transmission strategy only requires one extra time slot due to the broadcast transmission by the destination.
Similar to [2], we assume that each of the $L$ relays can decode the $m$-th source information if the source-relay channel satisfies $\log \left(1+\rho\left|h_{m r_{i}}\right|^{2}\right) \geq R$, where $h_{m r_{i}}$ is the coefficient for the wireless channel between the $m$-th source and the relay $r_{i}, R$ denotes the targeted data rate, and $\rho$ denotes the average received signal-to-noise ratio (SNR), which is assumed to be the same at each relay and the destination. Consider the index set of relays that can decode the transmission from all sources: $\left\{i:\left|h_{m r_{i}}\right|^{2} \geq \frac{2^{R}-1}{\rho}, \forall m \in\{1, \cdots, M\}\right\}$. The motivation for such a relay set will be discussed in the next section. Let $K$ denote the size of this qualified relay set. Note that it is assumed that there are a lot of relays, or idle nodes, in the network, which is valid for classical wireless sensor or cellular networks. For example, in a lecture hall, the total number of mobile users could be extremely large. Among these users, there are only a few ones making phone calls at a particular moment, whereas the rest of the users is idle. According to the proposed scheme, idle users can be used as relays and hence the number of relays, or idle users, can be quite large. Similar scenarios with a large number of relays can be found in those dense sensor networks. Through an error free signalling channel, the destination obtains the number of the qualified relays and determines the size of each data frame as $N=Q M$ where $Q=\left\lceil\frac{K+1}{M}\right\rceil$. Note that a node from the relaying set will be always qualified for the whole frame since quasi-static fading is considered here.

\section{B. Cooperative Transmission}

In contrast to the CMA strategies presented in [4], [9], the $M$ source nodes are not scheduled in a round-robin way. Instead, the source with the poorest connection to the destination transmits first. In other words, the $M$ source nodes are scheduled for transmission to satisfy

$$
\left|h_{1}\right|^{2} \leq\left|h_{2}\right|^{2} \leq \cdots \leq\left|h_{M}\right|^{2} .
$$

where $h_{m}$ denotes the coefficient of the channel between the $m$-th scheduled source and the destination. Similarly, the $K$ qualified relays are scheduled to satisfy

$$
\left|g_{1}\right|^{2} \leq\left|g_{2}\right|^{2} \leq \cdots \leq\left|g_{K}\right|^{2} .
$$

where $g_{k}$ denotes the coefficient for the channel between the destination and the relay scheduled at the $(K-k+1)$ th time $\operatorname{slot}^{1}$. The reason for such a schedule is to ensure that a source with a poor connection to the destination will receive better help from the relays than a source with a good connection. The details of the transmission protocol follow. The relay selection can be accomplished by using a centralized strategy, where the feedback of $L M$ complex-valued channels is required. To reduce the system overhead for scheduling sources and relays, the distributed selection strategy proposed in [15] can be applied. In particular, source transmissions can be scheduled in a distributed way by setting their backoff time proportional to the quality of their source-destination channels, whereas relay transmissions can be scheduled by

\footnotetext{
${ }^{1}$ Note that the relay associated with $g_{k}$ is not scheduled at the $k$-th time slot. The reason to have different sorting notation for the relays and sources is to simplify the development of analytical results, as shown later in this paper.
} 
setting their backoff time to be inversely proportional to the qualities of the relay-destination channels $g_{i}$. As a result, only the $K$ qualified relays are active and each of them needs to feedback one digit flag, which consumes much less system overhead than the centralized scheme. Similar to [3], [4], non-orthogonal transmissions have been adopted and hence medium access control protocols have to be designed carefully, which is beyond the scope of this paper.

The source with the channel coefficient $h_{1}$ is scheduled to transmit a message, $s_{1}(1)$, during the first time slot of each data frame. All $K$ qualified relays decode the message and store it in their memories. The destination receives

$$
y(1)=h_{1} s_{1}(1)+n(1),
$$

where $n(i)$ denotes the additive white Gaussian noise at the common destination. During the second time slot, the relay with the largest coefficient $g_{K}$ (the best relay-destination channel) is scheduled to help the user with the poorest channel condition and forward $s_{1}(1)$. At the same time, the source associated with $h_{2}$ is scheduled to transmit a new message $s_{2}(1)$. The destination receives the superimposed observation

$$
y(2)=h_{2} s_{2}(1)+g_{K} s_{1}(1)+n(2) .
$$

Although the remaining $(K-1)$ qualified relays also receive a mixture, the qualification criteria guarantee that each of them can decode $s_{2}(1)$ via simple successive decoding, since they were able to decode $s_{1}(1)$. Hence at the $n$-th time slot $(n=1, \ldots, N)$, the source with $h_{n^{\prime}}$ is scheduled to transmit a new message $s_{n^{\prime}}\left(\left\lceil\frac{n}{M}\right\rceil\right)$ where $n^{\prime}=(n \bmod M)$. At the same time, for $n \leq K$, the relay with $g_{K-n+1}$ will be scheduled to transmit its previous observation. For $n>K$, all qualified relays have been used and hence non-cooperative direct transmission will be adopted ${ }^{2}$.

As a result, the signal model for one data frame can be written as

$$
\mathbf{y}=\mathbf{H} \mathbf{s}+\mathbf{n},
$$

where $\quad \mathbf{y} \quad=\quad\left[\begin{array}{lll}y(1) & \cdots & y(N)\end{array}\right]^{T}, \quad \mathbf{s}$ $\left[\begin{array}{lllllll}s_{1}(1) & \cdots & s_{M}(1) & \cdots & s_{1}(Q) & \cdots & s_{M}(Q)\end{array}\right]^{T}$, $\mathbf{n}=\left[\begin{array}{lll}n(1) & \cdots & n(N)\end{array}\right]^{T}$ and the channel matrix is

$$
\mathbf{H}=\left[\begin{array}{ccccccc}
h_{1} & 0 & \ldots & \ldots & \ldots & \ldots & 0 \\
g_{K} & h_{2} & 0 & \vdots & \vdots & \vdots & 0 \\
\vdots & \ddots & \ddots & \vdots & \vdots & \vdots & \vdots \\
0 & 0 & g_{1} & h_{(K+1)^{\prime}} & 0 & \cdots & 0 \\
0 & 0 & 0 & 0 & h_{(K+2)^{\prime}} & 0 & 0 \\
\vdots & \vdots & \vdots & \vdots & \ddots & \ddots & \vdots \\
0 & 0 & 0 & 0 & 0 & 0 & h_{M}
\end{array}\right]_{N \times N}
$$

\footnotetext{
${ }^{2}$ Note that CSI between relays is needed for the proposed cooperative scheme. Such inter-relay CSI can be obtained in the following two ways. One is to ask each of the $K$ qualified relays to send training messages, which consumes extra system overhead. The other is to utilize the fact that each relay has some priori information for the received mixture, and apply the channel estimator based on so-called first-order statistics [16]. In such a case, dedicated training information is no longer needed, and the redundant structure of cooperative transmissions can be utilized to yield low-cost channel estimation.
}

An example for the signal model with $K=4$ and $M=3$ is shown as following

$$
\left[\begin{array}{l}
y(1) \\
y(2) \\
y(3) \\
y(4) \\
y(5) \\
y(6)
\end{array}\right]=\left[\begin{array}{cccccc}
h_{1} & 0 & 0 & 0 & 0 & 0 \\
g_{4} & h_{2} & 0 & 0 & 0 & 0 \\
0 & g_{3} & h_{3} & 0 & 0 & 0 \\
0 & 0 & g_{2} & h_{1} & 0 & 0 \\
0 & 0 & 0 & g_{1} & h_{2} & 0 \\
0 & 0 & 0 & 0 & 0 & h_{3}
\end{array}\right]\left[\begin{array}{l}
s_{1}(1) \\
s_{2}(1) \\
s_{3}(1) \\
s_{1}(2) \\
s_{2}(2) \\
s_{3}(2)
\end{array}\right] .
$$

\section{DIVERSITY-MULTIPLEXING TRADEOFF FOR THE PROPOSED CMA}

The aim of this section is to analytically evaluate the performance of the proposed cooperative protocol by calculating the diversity-multiplexing tradeoff. As can be seen from the signal model (1), the structure of the channel matrix $\mathbf{H}$ is not regular, which poses difficulties in obtaining an explicit expression for the outage probability and the tradeoff. In the following, we assume that the number of the qualified relays plus one, $K+1$, is an integer multiple of the number of sources $M$, which results in the following simpler channel matrix as

$$
\mathbf{H}=\left[\begin{array}{cccc}
h_{1} & 0 & 0 & 0 \\
g_{K} & h_{2} & 0 & 0 \\
\vdots & \ddots & \ddots & \vdots \\
0 & 0 & g_{1} & h_{M}
\end{array}\right]_{N \times N}
$$

Later in this paper, Monte-Carlo simulation results will be provided without assuming such a relationship between $K$ and $M$.

\section{A. Achievable diversity-multiplexing tradeoff}

First we recall the definition of the diversity gain and multiplexing gain as [17]

$$
d \triangleq-\lim _{\rho \rightarrow \infty} \frac{\log \left[P_{e}(\rho)\right]}{\log \rho}, \quad r \triangleq \lim _{\rho \rightarrow \infty} \frac{R(\rho)}{\log \rho},
$$

where $P_{e}$ is the probability of error of the maximum likelihood detector and $R$ is the data rate of a code. We say that a function $f(\rho)$ is said to be exponentially equal to $\rho^{d}$, denoted as $f(\rho) \doteq$ $\rho^{d}$, when $\lim _{\rho \rightarrow \infty} \frac{\log [f(\rho)]}{\log \rho}=d$. The following theorem provides an achievable diversity-multiplexing tradeoff for the proposed cooperative protocol conditioned on the size of the qualified relaying set.

Theorem 1: Assume that all addressed channels are i.i.d. quasi-static Rayleigh fading and the number of qualified relays is $K=Q M-1$. The following diversity-multiplexing tradeoff is achievable.

$$
d_{K}(r)=(1-r)+[K-(K+M) r]^{+},
$$

where $(x)^{+}$denote $\max \{x, 0\}$.

Proof: The details of the proof for this theorem will be provided in Section IV.

Recall that the number of the qualified relays $K$ is dynamically changing according to instantaneous channel realizations. Hence we would like to have the diversity-multiplexing tradeoff in terms of $L$. The important relationship between $K$ and 
the total number of relays $L$ is characterized in the following lemma

Lemma 2: Assume that all addressed channels are i.i.d quasi-static Rayleigh fading. Then,

$$
P(K=k) \doteq \rho^{-(L-k)(1-r)} .
$$

as $\rho \rightarrow \infty$.

Proof: Denote $h_{r_{i}}$ as the channel which has the smallest absolute value among the set containing the $M$ channels between the $i$-th relay and $M$ sources. Consider the set $\left\{h_{r_{1}}, \ldots, h_{r_{L}}\right\}$ which collects the $L$ worst source-relay channels. Under the Rayleigh fading assumption, these $L$ variables $\left|h_{r_{i}}\right|^{2}$ are i.i.d with density function

$$
f(x)=M e^{-M x} .
$$

Consider the $L$ channels ordered as $\left|h_{r_{(1)}}\right|^{2} \geq \ldots \geq\left|h_{r_{(L)}}\right|^{2}$. The event that the size of the qualified set is $K=k$ means $\left|h_{r_{(k)}}\right|^{2} \geq \gamma \geq\left|h_{r_{(k+1)}}\right|^{2}$, where $\gamma=\frac{2^{R}-1}{\rho}$. By using order statistics, the probability that the size of the qualified relay set is $K=k$ can be expressed as [14]

$$
P(K=k)=\frac{L !}{(L-k) !(k) !}\left[1-e^{-M \gamma}\right]^{L-k}\left[e^{-M \gamma}\right]^{k},
$$

where $\gamma=\frac{2^{R}-1}{\rho} \doteq \rho^{r-1}$, as $\rho \rightarrow \infty$. When $\rho \rightarrow \infty$, the use of the exponential expansion yields

$$
P(K=k) \doteq \frac{L !}{(L-k) !(k) !}(M \gamma)^{L-k} \doteq \rho^{-(1-r)(L-k)}(.6)
$$

Combining Theorem 1 and Lemma 2, the overall outage probability can be expressed as ${ }^{3}$

$$
\begin{aligned}
P(\mathcal{O}) & =\sum_{k=0}^{L} P(\mathcal{O} \mid K=k) P(K=k) \\
& \doteq \sum_{k=0}^{L} \rho^{-(L-k+1)(1-r)-[k-(k+M) r]^{+}} \doteq \rho^{-d(r)} .
\end{aligned}
$$

where $d(r)=(1-r)+[L-(L+M) r]^{+}$and the last relationship follows from the fact that the error probability with $K=L$ is the dominant factor. For a fixed data rate, (7) shows that the proposed cooperative multiple access scheme can achieve the maximum possible diversity gain $L+1$. Thus, although each user only uses a fraction of the available relays, this full diversity gain is still achieved with the help of the opportunistic scheduling. Rewrite the achievable diversitymultiplexing tradeoff as

$$
d(r)=\left\{\begin{array}{ll}
(L+1)\left(1-r-\frac{M}{L+1} r\right), & \text { if } 0 \leq r \leq \frac{L}{L+M} \\
1-r, & \text { if } \frac{L}{L+M}<r \leq 1
\end{array} .\right.
$$

The optimal tradeoff for the traditional multiple-input singleoutput (MISO) scheme can be expressed as [17]

$$
d_{M I S O}(r)=(L+1)(1-r), \quad \text { if } 0 \leq r \leq 1 .
$$

\footnotetext{
${ }^{3}$ Note that the diversity-multiplexing tradeoff in Theorem 1 is obtained with the assumption $K=Q M$, which imposes a constraint on the realization of $K$ that is not considered here. For simulation results, this assumption about $K$ will not be used
}

As the number of available relays $L$ becomes large for a fixed number of sources $M$, the tradeoff achievable for the proposed transmission scheme approximates the optimal MISO tradeoff, $d(r) \rightarrow d_{M I S O}(r)$.

On the other hand, the diversity-multiplexing tradeoff achieved by the CMA schemes in [4], [9] can be written as

$$
d_{C M A}(r)=M(1-r), \quad \text { if } 0 \leq r \leq 1 .
$$

The advantage of the proposed relay-assisted CMA is its capability to exploit the relay nodes as an extra dimension and enhance the robustness of reception, whereas the diversity gain of the schemes in [4], [9] is constrained by the number of source nodes. Such a capability to utilize relays is particularly important for a network where the number of idle users is much larger than the number of active ones. However, for a large value of the multiplexing gain $r$, the DMT achieved by the relay-assisted CMA is worse than those using the source nodes only in [4], [9].

Obviously the opportunistic relaying scheme in [15] can be straightforwardly extended to multiple access channels by applying the time sharing approach, which yields the diversitymultiplexing tradeoff $d_{o s}(r)=L(1-2 r), 0 \leq r \leq 0.5$ [18]. Another comparable scheme for multiple access transmissions has been proposed in [6]. To combat co-channel interference, the scheme in [6] requires $2 M$ time slots to transmit $M$ source messages, which results in the fact that its achievable multiplexing gain is 0.5 at most. Comparing to these two cooperative schemes, the proposed transmission scheme achieves larger diversity gains for the multiplexing gains $1 \geq r \geq 0.5$.

\section{B. Numerical Results}

The analytical results developed in the previous section require the assumptions $K=Q M-1$ and $K=L$. In this section, we provide some numerical results based on MonteCarlo simulations that do not rely on these assumptions.

In the first experiment, the outage performance of the proposed CMA scheme is compared with the superimposed modulation based CMA in [4], [9] as well as the noncooperative scheme. The number of source nodes is set to $M=2$ and the number of relays is fixed as $L=5$. The target data rate is set to $R=4$ and $R=8$ bits per channel use (BPCU). By increasing the target data rate, the performances of all studied schemes decrease as shown in Fig. 1. We further observe that the proposed CMA scheme achieves smaller outage probabilities compared with the two comparable schemes for all SNR. The reason for this performance gain is that the comparable cooperative schemes do not exploit the available relays and their diversity gain is constrained by the number of sources.

In the second experiment, the outage probability achieved by the proposed scheme is shown in Fig. 2 as a function of SNR and with different numbers of relay candidates, $L$. The number of source nodes is set to $M=2$. As can be seen from the figure, the robustness of the multiple access transmission can be improved by increasing the number of the relays. As indicated by Theorem 1, increasing the number of relays not only increases the diversity gain, but also improve the spectral 
efficiency of the proposed CMA scheme since the tradeoff achievable for the proposed scheme is approaching the optimal MISO upper bound.

\section{Proof of Theorem 1}

As discussed in [17], the error probability of the maximum likelihood detector can be tightly bounded by the outage probability at high SNR, and hence the outage probability will be used as the criterion for performance evaluation in this paper. Since the multi-user scenario is considered, the outage event can be defined as [8]

$$
\mathcal{O} \triangleq \bigcup_{\mathcal{A}} \mathcal{O}_{\mathcal{A}}
$$

and the outage probability of the cooperative network can be written as

$$
P(\mathcal{O})=P\left(\bigcup_{\mathcal{A}} \mathcal{O}_{\mathcal{A}}\right) \leq \sum_{\mathcal{A}} P\left(\mathcal{O}_{\mathcal{A}}\right),
$$

where

$$
\mathcal{O}_{\mathcal{A}} \triangleq\left\{\mathcal{I}\left(\mathbf{s}_{\mathcal{A}} ; \mathbf{y} \mid \mathbf{s}_{\mathcal{A}^{c}}, \mathbf{H}=H\right)<Q \sum_{i \in \mathcal{A}} R_{i}\right\},
$$

$R_{i}$ denotes the target data rate for the $i$-th source, $\mathcal{A} \subseteq$ $\{1, \ldots, M\}$ and $|\mathcal{A}|$ is denoted as the number of users in $\mathcal{A}$. Note that the symmetric tradeoff is of interest in this paper, which means $|\mathcal{A}| R=\sum_{i \in \mathcal{A}} R_{i}$. From the definition, the mutual information for each outage event can be written as

$$
\mathcal{I}_{\mathcal{A}}=\log \operatorname{det}\left[\mathbf{I}_{N}+\rho \mathbf{H} \boldsymbol{\Gamma}_{\mathcal{A}} \mathbf{H}^{H}\right] \text {, }
$$

where $\mathcal{I}_{\mathcal{A}} \triangleq \mathcal{I}\left(\mathbf{s}_{\mathcal{A}} ; \mathbf{y} \mid \mathbf{s}_{\mathcal{A}^{c}}, \mathbf{H}=H\right)$, and $\boldsymbol{\Gamma}_{\mathcal{A}}$ is formed by removing the columns of the identity matrix $\mathbf{I}_{N}$ corresponding to columns of $\mathbf{H}$ associated with the users in $\mathcal{A}^{c}$. Take $\mathcal{A}=$ $\{i\}$ as an example. The $Q$ diagonal elements of $\Gamma_{\mathcal{A}}$ are ones, $\Gamma_{\mathcal{A}}[(q-1) Q+i,(q-1) Q+i]=1$ for $q \in\{1, \ldots, Q\}$, and all other elements are zero.

According to the elements contained in the set $\mathcal{A}$, the outage events can be classified into three events.

- The first event, denoted by $\mathcal{O}_{1}$, is an event where one user is in outage (i.e. $|\mathcal{A}|=1$ ). Furthermore, $f(\rho)$ is said to be exponentially smaller than $\rho^{d}$, denoted as $f(\rho) \dot{\leq} \rho^{d}$, when $\lim _{\rho \rightarrow \infty} \frac{\log [f(\rho)]}{\log \rho} \leq d$. The following lemma provides an upper bound for the outage probability of this type of events.

Lemma 3: Assume that all channels are i.i.d. Rayleigh fading and the number of relays is $K=Q M-1$. The outage probability for the event $\mathcal{O}_{1}$ is upper bounded as

$$
P\left(\mathcal{O}_{1}\right) \dot{\leq} \rho^{-d_{\mathcal{O}_{1}}(r)}
$$

where $d_{\mathcal{O}_{1}}(r)=1-r+(K-(K+M) r)^{+}$.

Proof: See Appendix.

- The second outage event, denoted as $\mathcal{O}_{2}$, is the event where the overall sum rate is in outage (i,e, the set $\mathcal{A}$ contains all $M$ users, $|\mathcal{A}|=M)$. The following lemma provides an upper bound for the outage probability associated with such an event.
Lemma 4: Assume that all addressed channels are i.i.d. Rayleigh fading and the number of the relays is $K=$ $Q M-1$. The outage probability for the event $\mathcal{O}_{2}$ can be upper bounded as

$$
P\left(\mathcal{O}_{2}\right) \dot{\leq} \rho^{-d_{\mathcal{O}_{2}}(r)},
$$

where $d_{\mathcal{O}_{2}}(r)=M(1-r)+(K-(K+1) r)^{+}$.

Proof: According to the signal model, the sum rate can be written as

$$
\mathcal{I}_{\mathcal{A}}=\log \operatorname{det}\left[\mathbf{I}_{N}+\rho \mathbf{H} \mathbf{H}^{H}\right] .
$$

It is easy to show that $\mathbf{H} \mathbf{H}^{H}$ is a tridiagonal matrix, and hence $\left[\mathbf{I}_{N}+\mathbf{H} \mathbf{H}^{H}\right]$ is also a tridiagonal matrix. According to [19], the determinant of a tridiagonal matrix can be obtained iteratively as

$$
D_{n}=\left[1+\rho\left(x_{n}+y_{n-1}\right)\right] D_{n-1}-\rho^{2} x_{n} y_{n-1} D_{n-2} \text {. }
$$

where $D_{n}=\operatorname{det}\left[\mathbf{I}_{n}+\mathbf{H}_{n-1} \mathbf{H}_{n-1}^{H}\right], \mathbf{H}_{n}$ is the $n \times n$ top-left submatrix from $\mathbf{H}$, and $x_{n}$ and $y_{n}$ are the $n$-th element on the principle diagonal and subdiagonal of $\mathbf{H}$, respectively. By using such a property, we can obtain the following inequality

$$
\mathcal{I}_{\mathcal{A}} \geq \log \left(\left[\prod_{i=1}^{M}\left(1+\rho\left|h_{i}\right|^{2}\right)\right]^{Q}+\left[\prod_{i=1}^{K} \rho\left|g_{i}\right|^{2}\right]\right),
$$

whose proof can be accomplished by following similar steps in [20]. Hence the outage probability can be upper bounded as

$$
\begin{aligned}
P\left(\mathcal{I}_{\mathcal{A}}<Q M R\right) & \leq P\left\{\left(z_{1}+z_{2}\right)<2^{Q M R}\right\} \\
& \leq P\left\{z_{1}<2^{Q M R}\right\} P\left\{z_{2}<2^{Q M R}\right\} \\
& \doteq \frac{1}{\rho^{M-M r}} \cdot \frac{1}{\rho^{K-(K+1) r}}
\end{aligned}
$$

where $z_{1}=\left[\prod_{i=1}^{M}\left(1+\rho\left|h_{i}\right|^{2}\right)\right]^{Q}, z_{2}=\left[\prod_{i=1}^{K} \rho\left|g_{i}\right|^{2}\right]$, and the last inequality follows from the proof for Lemma 3.

- The third type of outage events, denoted as $\mathcal{O}_{3}$, corresponds to the case where the set $\mathcal{A}$ contains more than one user, and less than $M$ users, $1<|\mathcal{A}|<M$. Obtaining a closed-form expression for the probability for $\mathcal{O}_{3}$ is difficult, and we only prove that the probability of $\mathcal{O}_{3}$ is upper bounded by the probability of $\mathcal{O}_{1}$. The following lemma will be needed for the development of $P\left(\mathcal{O}_{3}\right)$.

Lemma 5: Consider a $(P+1) \times P$ complex-valued matrix, denoted as $\boldsymbol{\Theta}_{P}$, whose $(m, m)$-th element is $h_{m}$ and its $(m+1, m)$-th element is $g_{m}$ for $m \in\{1, \ldots, P\}$. The remaining elements of this matrix are zero. The following inequality holds

$$
\operatorname{det}\left\{\mathbf{I}_{P}+\rho \boldsymbol{\Theta}_{P}^{H} \boldsymbol{\Theta}_{P}\right\} \geq \prod_{i=1}^{P} \rho\left|h_{i}\right|^{2}+\prod_{j=1}^{P}\left(1+\rho\left|g_{j}\right|^{2}\right) .
$$

Proof: See Appendix.

By using this lemma, we can have the following result about the probability of $\mathcal{O}_{3}$ and $\mathbf{O}_{1}$. 
Lemma 6: Assume that all wireless channels are i.i.d. Rayleigh fading and the number of relay is $K=Q M-1$. The probability of $\mathcal{O}_{3}$ can be upper bounded as

$$
P\left(\mathcal{O}_{3}\right) \dot{\leq} P\left(\mathcal{O}_{1}\right) \dot{\leq} \rho^{-d_{\mathcal{O}_{1}}(r)} .
$$

Proof: See Appendix.

Of the three events, the probability of $\mathcal{O}_{1}$ dominates. As a result, the overall outage probability can be upper bounded as

$$
P(\mathcal{O}) \leq \sum_{\mathcal{A}} P\left(\mathcal{O}_{\mathcal{A}}\right) \doteq \rho^{-d_{\mathcal{O}_{1}}(r)},
$$

proving Theorem 1 .

\section{CONCLUSION}

In this paper, we have proposed a spectrally efficient cooperative transmission protocol for multiple access scenarios. By carefully scheduling the multiple sources and relays' transmission, the full diversity gain can be achievable for each user although only a fraction of relays is utilized by each user. An achievable diversity-multiplexing tradeoff (DMT) was developed. With a large number of relays, the DMT achieved by the proposed scheme can approach the optimal multiple-input single-input upper bound. Both analytical and numerical results demonstrated that the proposed protocol can outperform comparative schemes that employ only source cooperation in most conditions.

\section{APPENDIX}

Proof of Lemma 3: The set of the users $\mathcal{A}$ corresponding to the outage event $\mathcal{O}_{1}$ can be further divided into two types which will be studied separately in the following two subsections.

\section{A. The event with $\mathcal{A}=\{n\}$ and $1 \leq n \leq M-1$}

Recall that such a outage event corresponds the case where all users' information can be decoded correctly expect the $n$-th user's message, which means that the mutual information can be written as

$$
\mathcal{I}_{\mathcal{A}}=\log \prod_{i=1}^{Q}\left[1+\rho\left(\left|h_{n}\right|^{2}+\left|g_{i M-n}\right|^{2}\right)\right] .
$$

Hence the outage probability can be upper bounded as

$$
\begin{array}{r}
P\left(\mathcal{I}_{\mathcal{A}}<Q R\right)=P\left\{\prod_{i=1}^{Q}\left[1+\rho\left(\left|h_{n}\right|^{2}+\left|g_{i M-n}\right|^{2}\right)\right]<2^{Q R}\right\} \\
\leq P\left\{\left(\rho\left|h_{n}\right|^{2}\right)^{Q}+\prod_{i=1}^{Q}\left[1+\rho\left|g_{i M-n}\right|^{2}\right]<2^{Q R}\right\} \\
\leq P\left\{\rho\left|h_{n}\right|^{2}<2^{R}\right\} P\left\{\prod_{i=1}^{Q}\left[1+\rho\left|g_{i M-n}\right|^{2}\right]<2^{Q R}\right\} .
\end{array}
$$

Denote $y_{n}=\left|h_{n}\right|^{2}$ and $x_{i}=\left|g_{i}\right|^{2}$. The factor $P\left\{\rho y_{n}<2^{R}\right\}$ can be easily analyzed as following. According to the adopted transmission scheduler, $y_{n}$ is the $n$-th smallest among $M$ i.i.d. exponentially distributed variables. Hence, its probability density function is

$$
f_{y_{n}}(y)=\frac{M !}{(n-1) !(M-n) !} e^{-(M-n+1) y}\left[1-e^{-y}\right]^{n-1} .
$$

And its outage probability can be written as

$$
P\left\{\rho y_{n}<2^{R}\right\}=\int_{0}^{\frac{2^{R}}{\rho}} \frac{M ! e^{-(M-n+1) y}}{(n-1) !(M-n) !}\left[1-e^{-y}\right]^{n-1} d y
$$

Applying $R=r \log \rho$, we obtain $\frac{2^{R}}{\rho}=\rho^{-(1-r)}$. Hence the facts of $r \leq 1$ and $\rho \rightarrow \infty$ yields $\rho^{-(1-r)}<<1$, and thus

$$
P\left\{\rho\left|h_{n}\right|^{2}<2^{R}\right\} \doteq \int^{\rho^{-(1-r)}} \frac{M ! y^{n-1} d y}{(n-1) !(M-n) !} \doteq \frac{1}{\rho^{n(1-r)}}
$$

where the exponential expansion is used to obtain the approximation.

Define $\mathcal{I}=\ln \prod_{i=1}^{Q}\left[1+\rho x_{i M-n}\right]$. The density function of $\mathcal{I}$ will be first studied to obtain the outage probability $P\{\mathcal{I}<Q R \ln 2\}$. Recall that the density of the mutual information can be written as

$$
q(\mathcal{I})=\mathcal{E}_{\mathbf{g}}\left\{\delta\left(\mathcal{I}-\prod_{i=1}^{Q}\left[1+\rho x_{i M-n}\right]\right)\right\},
$$

which contains a non-analytical Dirac delta function, see [21]. To find the outage probability, the delta function has to be removed from the expression of $q(\mathcal{I})$. Recall the following property of the delta function:

$$
\int_{b}^{a} \delta\left(\psi_{n}-\psi\right) \delta\left(\psi-\psi_{m}\right) d \psi=\delta\left(\psi_{n}-\psi_{m}\right)
$$

which can be used to separate $x_{i}$ in the delta function. Hence we have

$$
\begin{aligned}
q(\mathcal{I})=e^{\mathcal{I}} \int_{0}^{\infty} & \cdots \int_{0}^{x_{(Q-1) M-n}} \delta\left(e^{\mathcal{I}}-\prod_{i=1}^{Q}\left(1+\rho x_{i M-n}\right)\right) \\
& \times f\left(x_{M-n}, \cdots, x_{Q M-n}\right) d x_{M-n} \cdots d x_{Q M-n},
\end{aligned}
$$

where the joint density function of the $Q$ ordered variables can be written as [14]

$$
\begin{array}{r}
f\left(x_{M-n}, \cdots, x_{Q M-n}\right)=K !\left(\prod_{i=1}^{Q} f\left(x_{r_{i}}\right)\right) \frac{\left[F\left(x_{r_{1}}\right)\right]^{M-n-1}}{(M-n-1) !} \\
\times \frac{\left[1-F\left(x_{r_{Q}}\right)\right]^{n-1}}{(n-1) !} \prod_{i=2}^{Q} \frac{\left[F\left(x_{r_{i}}\right)-F\left(x_{r_{i-1}}\right)\right]^{M-1}}{(M-1) !},
\end{array}
$$

where $f(x)$ denotes the probability density function (PDF) $f(x)=e^{-x}$ and $F(x)$ is the cumulative distribution function (CDF) $F(x)=1-e^{-x}$. Since an upper bound to the outage probability is of interest, the following bound is employed to simplify the expression of the joint density function as

$$
\begin{aligned}
& f\left(x_{M-n}, x_{2 M-n}, \cdots, x_{Q M-n}\right) \leq \\
& K ! \prod_{i=1}^{Q} f\left(x_{r_{i}}\right) \frac{\left[F\left(x_{r_{1}}\right)\right]^{M-n-1}}{(M-n-1) !} \prod_{i=2}^{Q} \frac{\left[F\left(x_{r_{i}}\right)\right]^{M-1}}{(M-1) !},
\end{aligned}
$$

where the inequality follows from the fact that the CDF is always positive. Furthermore, since $f\left(x_{i}\right)=e^{-x_{i}} \leq 1$, we 
have $\prod_{i=1}^{Q} f\left(x_{r_{i}}\right) \leq 1$. Together with the fact that $1-e^{-x} \leq x$ for $x \geq 0$, we have

$$
\begin{array}{r}
f\left(x_{M-n}, \cdots, x_{Q M-n}\right) \leq K ! \frac{\left[x_{M-n}\right]^{M-n-1}}{(M-n-1) !} \prod_{i=2}^{Q} \frac{x_{i M-n}^{M-1}}{(M-1) !} \\
=f^{\prime}\left(x_{M-n}, \cdots, x_{Q M-n}\right)
\end{array}
$$

where $C_{1}=\frac{K !}{[(M-1) !]^{Q-1}(M-n-1) !}$ and $f^{\prime}=$ $C_{1}\left\{\prod_{i=2}^{Q} x_{i M-n}\right\}^{M-1} x_{M-n}^{M-n-1}$. To further simplify the development, the integration region of the expectation in (15) is extended to the entire positive plane

$$
\begin{aligned}
q(\mathcal{I}) \leq & C_{1} e^{\mathcal{I}} \int_{0}^{\infty} \cdots \int_{0}^{\infty} \delta\left(e^{\mathcal{I}}-\prod_{i=1}^{Q}\left(1+\rho x_{i M-n}\right)\right) \\
& \times f^{\prime}\left(x_{M-n}, \cdots, x_{Q M-n}\right) d x_{M-n} \cdots d x_{Q M-n},
\end{aligned}
$$

since the simplified joint density function is always positive over the addressed region.

In the following, we will focus on how to remove the delta function out of the density function. First we obtain the expression as shown in the top of the next page. where the following property of the delta function is used

$$
\int_{b}^{a} \delta\left(\psi_{n}-\psi\right) \delta\left(\psi-\psi_{m}\right) d \psi=\delta\left(\psi_{n}-\psi_{m}\right)
$$

Again using the property (16) we obtain the equation shown in (17). Recall another property of the delta function as

$$
\int_{D} \delta(x-\psi) f(x) d x=\left\{\begin{array}{cc}
f(\psi) & \psi \in D \\
0 & \text { otherwise }
\end{array} .\right.
$$

Together with the high SNR assumption, we can finally remove the delta function from the density function

$q(\mathcal{I}) \leq \frac{e^{\mathcal{I}}}{\rho^{Q}} \int_{1}^{e^{\mathcal{I}}} \int_{1}^{\psi_{1}} \cdots \int_{1}^{\psi_{Q-2}} \Psi\left(\psi_{1}, \ldots, \psi_{Q-1}, \rho\right) \prod_{i=1}^{Q-1} \frac{1}{\psi_{i}} d \psi_{i}$,

where

$\Psi\left(\psi_{1}, \ldots, \psi_{Q-1}, \rho\right)=f^{\prime}\left(\frac{e^{\mathcal{I}}}{\rho \psi_{1}}, \frac{\psi_{1}}{\rho \psi_{2}}, \cdots, \frac{\psi_{Q-2}}{\rho \psi_{Q-1}}, \frac{\psi_{Q-1}}{\rho}\right)$.

Substituting the simplified density function, we can obtain

$$
\begin{array}{r}
\Psi\left(\psi_{1}, \ldots, \psi_{Q-1}, \rho\right)=f^{\prime}\left(\frac{e^{\mathcal{I}^{\prime}}}{\rho \psi_{1}}, \frac{\psi_{1}}{\rho \psi_{2}}, \cdots, \frac{\psi_{Q-2}}{\rho \psi_{Q-1}}, \frac{\psi_{Q-1}}{\rho}\right) \\
\leq \frac{C_{1}}{\rho^{Q(M-1)-n}} e^{(M-n-1) \mathcal{I}} \psi_{1}^{n} .
\end{array}
$$

Interestingly, only one variable $\psi_{1}$ is left in the joint density function, which shall simplify the following development significantly. By using this simplified result, the density of the mutual information at high SNR can be upper bounded as

$$
\begin{array}{r}
q(\mathcal{I})=\frac{e^{\mathcal{I}}}{\rho^{Q}} \int_{1}^{e^{\mathcal{I}}} \int_{1}^{\psi_{1}} \cdots \int_{1}^{\psi_{Q-2}} \frac{C_{1} e^{(M-n-1) \mathcal{I}}}{\rho^{Q(M-1)-n}} \psi_{1}^{n} \prod_{i=1}^{Q-1} \frac{1}{\psi_{i}} d \psi_{i}, \\
=\frac{C_{1} e^{(M-n) \mathcal{I}}}{\rho^{Q M-n}} \int_{1}^{e^{\mathcal{I}}} \psi_{1}^{n} \int_{1}^{\psi_{1}} \cdots \int_{1}^{\psi_{Q-2}} \prod_{i=1}^{Q-1} \frac{1}{\psi_{i}} d \psi_{i}
\end{array}
$$

which can be written as

$$
\begin{aligned}
q(\mathcal{I}) & =\frac{C_{1} e^{(M-n) \mathcal{I}}}{\rho^{Q M-n}} \int_{0}^{\mathcal{I}} e^{n \phi_{1}} \int_{0}^{\phi_{1}} \cdots \int_{0}^{\phi_{Q-2}} \prod_{i=1}^{Q-1} d \phi_{i} \\
& =\frac{C_{1} e^{(M-n) \mathcal{I}}}{\rho^{Q M-n}(Q-2) !} \int_{0}^{\mathcal{I}} e^{n \phi_{1}} \phi_{1}^{(Q-2)} d \phi_{1} \\
& =\frac{C_{1} e^{(M-n) \mathcal{I}}}{\rho^{Q M-n}}\left(\frac{1}{(-n)^{Q-1}}-e^{n \mathcal{I}} \sum_{k=0}^{Q-2} \frac{\mathcal{I}^{k}}{k !(-n)^{Q-k-1}}\right) .
\end{aligned}
$$

where $\phi_{i}=\ln \psi_{i}$ and the last equality follows from $\mathrm{Eq}$ (3.351.1) in [22]. Given the density function of the mutual information, the outage probability can be expressed as the integral of the density function

$$
\begin{aligned}
P(\mathcal{I}<Q R \ln 2) \leq & \int_{0}^{Q R \ln 2} q(\mathcal{I}) d \mathcal{I} \\
= & \int_{0}^{Q R \ln 2} \frac{C_{1} e^{(M-n) \mathcal{I}}}{\rho^{Q M-n}} \frac{1}{(-n)^{Q-1}} d \mathcal{I} \\
& -\sum_{k=0}^{Q-2} \int_{0}^{Q R \ln 2} \frac{C_{1} e^{M \mathcal{I}}}{\rho^{Q M-n}} \frac{\mathcal{I}^{k}}{k !(-n)^{Q-k-1}} d \mathcal{I} .
\end{aligned}
$$

Interestingly the second integral at the right hand of the previous equation can be solved again using $\mathrm{Eq(3.351.1)}$ in [22] as

$$
P(\mathcal{I}<Q R \ln 2) \leq \frac{C_{1} e^{(M-n) Q R \ln 2}}{\rho^{Q M-n}(-n)^{Q-1}}-\sum_{k=0}^{Q-2} \frac{C_{1}}{(-n)^{Q-k-1}}
$$

$\times \frac{1}{\rho^{Q M-n} k !}\left(\frac{1}{(-M)^{k+1}}-e^{M Q R \ln 2} \sum_{j=0}^{k} \frac{(Q R \ln 2)^{j}}{j !(-M)^{k+1-j}}\right)$.

Recall that the diversity-multiplexing tradeoff can be obtained by substituting $R=r \log \rho$ into the outage probability. By using the relationship between $r$ and $R$, we first have

$$
Q R \ln 2=\frac{Q r \log \rho}{\log e}=\ln \rho^{Q r} .
$$

Following similar steps in [9], it can be shown that

$$
\frac{C_{1} e^{(M-n) Q R \ln 2}}{\rho^{Q M-n}} \frac{1}{(-n)^{Q-1}} \doteq \frac{\rho^{(M-n) Q r}}{\rho^{Q M-n}} \frac{1}{(-1)^{Q-1}},
$$

and

$$
\begin{aligned}
& -\sum_{k=0}^{Q-2} \frac{C_{1}}{\rho^{Q M-n} k !(-n)^{Q-k-1}}\left(\frac{1}{(-M)^{k+1}}\right. \\
& \left.-e^{M Q R \ln 2} \sum_{j=0}^{k} \frac{(Q R \ln 2)^{j}}{j !(-M)^{k+1-j}}\right) \doteq \frac{\rho^{M Q r}}{\rho^{Q M-n}} .
\end{aligned}
$$

Obviously the latter is dominant at high SNR, and hence the outage probability can be upper bounded as

$$
P(\mathcal{I}<Q R \ln 2) \leq \frac{\rho^{M Q r}}{\rho^{Q M-n}}, \forall n \in\{1, \cdots, M-1)(22)
$$

And the outage probability for $\mathcal{O}_{\mathcal{A}}$ can be expressed as

$$
\begin{aligned}
P\left(\mathcal{I}_{\mathcal{A}}<Q R\right) & =P\left(\rho\left|h_{n}\right|^{2}<2^{R}\right) P(\mathcal{I}<Q R \ln 2) \\
& \leq \frac{\rho^{(n+M Q) r}}{\rho^{Q M}}, \forall n \in\{1, \cdots, M-1\}
\end{aligned}
$$




$$
\begin{aligned}
q(\mathcal{I}) \leq & e^{\mathcal{I}} \underbrace{\int_{1}^{e^{\mathcal{I}}} \int_{1}^{\psi_{1}} \cdots \int_{x_{M-n}, \cdots, x_{Q M-n}}^{\psi_{Q-2}}}_{\psi_{1}, \cdots, \psi_{Q-1}} \underbrace{\int_{0}^{\infty} \cdots \int_{0}^{\infty} \delta}\left(e^{\mathcal{I}}-\left[1+\rho x_{M-n}\right] \psi_{1}\right) \delta\left(\psi_{1}-\left[1+\rho x_{2 M-n}\right] \psi_{2}\right) \cdots \\
& \times \delta\left(\psi_{Q-1}-\left[1+\rho x_{Q M-n}\right]\right) f^{\prime}\left(x_{M-n}, \cdots, x_{Q M-n}\right) d x_{M-n} \cdots d x_{Q M-n}, d \psi_{1} \cdots d \psi_{N-1}, \\
q(\mathcal{I}) \leq & e^{\mathcal{I}} \int \cdots \int \frac{1}{\rho \psi_{1}} \delta\left(x_{M-n}-\frac{e^{\mathcal{I}}-\psi_{1}}{\rho \psi_{1}}\right) \frac{1}{\rho \psi_{2}} \delta\left(x_{2 M-n}-\frac{\psi_{1}-\psi_{2}}{\rho \psi_{2}}\right) \cdots \\
& \times \frac{1}{\rho} \delta\left(x_{Q M-n}-\frac{\psi_{Q-1}-1}{\rho}\right) f^{\prime}\left(x_{M-n}, \cdots, x_{Q M-n}\right) d x_{M-n} \cdots d x_{Q M-n}, d \psi_{1} \cdots d \psi_{N-1} .
\end{aligned}
$$

\section{B. The event with $\mathcal{A}=\{M\}$}

The event with the case $\mathcal{A}=\{M\}$ is different to the cases with $\mathcal{A}=\{i, i \neq M\}$. According to the proposed transmission protocol, only $(Q-1)$ relays will be used to help the $M$-th user's information whereas $Q$ relays are scheduled to help each of the rest of the users. As a result, the expression of the mutual information can be written as

$$
\begin{array}{r}
\mathcal{I}_{\mathcal{A}}=\log \left\{\left[1+\rho\left|h_{M}\right|^{2}\right] \prod_{i=2}^{Q}\left[1+\rho\left(\left|h_{M}\right|^{2}+\left|g_{i M-M}\right|^{2}\right)\right]\right\} \\
\leq \log \left\{\left[1+\rho\left|h_{M}\right|^{2}\right]^{Q}+\prod_{i=2}^{Q}\left[1+\rho\left|g_{i M-M}\right|^{2}\right]\right\} .
\end{array}
$$

Comparing with the expression of $\mathcal{I}$ in (14), the number of the relay-destination channels is reduced to $Q-1$. By using such an approximation, we can upper bound the outage probability as

$$
\begin{aligned}
P\left(\mathcal{I}_{\mathcal{A}}<Q R\right) \leq & P\left\{\left[1+\rho\left|h_{M}\right|^{2}\right]^{Q}<2^{Q R}\right\} \\
& \times P\left\{\prod_{i=2}^{Q}\left[1+\rho\left|g_{i M-n}\right|^{2}\right]<2^{Q R}\right\} .
\end{aligned}
$$

As discussed previously, it can show that $P\left\{\left[1+\rho\left|h_{M}\right|^{2}\right]^{Q}<2^{Q R}\right\} \dot{\dot{\leq}} \frac{1}{\rho^{M-M r}} . \quad$ Again define $\mathcal{I}=\ln \prod_{i=2}^{Q}\left[1+\rho\left|g_{i M-n}\right|^{2}\right]$. The second probability can be evaluated by using the steps similar to the previous section. Here we only point out the different and key steps. First the mutual information can be shown as

$$
q(\mathcal{I})=\frac{e^{\mathcal{I}}}{\rho^{Q-1}} \int_{1}^{e^{\mathcal{I}}} \int_{1}^{\psi_{1}} \cdots \int_{1}^{\psi_{Q-3}} \Psi \prod_{i=1}^{Q-2} \frac{1}{\psi_{i}} d \psi_{i},
$$

where

$$
\Psi=f^{\prime}\left(\frac{e^{\mathcal{I}}}{\rho \psi_{1}}, \frac{\psi_{1}}{\rho \psi_{2}}, \cdots, \frac{\psi_{Q-3}}{\rho \psi_{Q-2}}, \frac{\psi_{Q-2}}{\rho}\right) .
$$

From the order statistics,

$$
\begin{array}{r}
f\left(x_{M}, \cdots, x_{(Q-1) M}\right)=K ! \prod_{i=1}^{Q-1} f\left(x_{i M}\right) \prod_{i=2}^{Q-1} \frac{\left[F\left(x_{M}\right)\right]^{M-1}}{(M-1) !} \\
\times \frac{\left[F\left(x_{i M}\right)-F\left(x_{i M-M}\right)\right]^{M-1}}{(M-1) !} \frac{\left[1-F\left(x_{Q M-M}\right)\right]^{M-1}}{(M-1) !}
\end{array}
$$

Since $f\left(x_{i}\right)=e^{-x_{i}} \leq 1$, we have $\prod_{i=1}^{Q} f\left(x_{r_{i}}\right) \leq 1$. Also $F\left(x_{i}\right) \leq 1$ and $1-F\left(x_{i}\right) \leq 1$ and $F\left(x_{i+1}\right)-F\left(x_{i}\right) \leq x_{i+1}$. So the simplified joint density function can be expressed as

$$
\begin{array}{r}
f\left(x_{M-n}, \cdots, x_{Q M-n}\right) \leq f^{\prime}\left(x_{M-n}, \cdots, x_{Q M-n}\right) \\
=C_{2}\left\{\prod_{i=1}^{Q-1} x_{i M}\right\}^{M-1},
\end{array}
$$

where $C_{2}=\frac{K !}{[(M-1) !]^{Q}}$. By using this simplified expression, we obtain

$$
\Psi\left(\psi_{1}, \ldots, \psi_{Q-2}, \rho\right) \leq \frac{C_{1}}{\rho^{(Q-1)(M-1)}} e^{(M-1) \mathcal{I}},
$$

which is no longer a function of $\psi_{i}$. Hence the density of the mutual information is simpler compared with the case $\mathcal{A}=$ $\{i, i \neq M\}$ and can be written as

$$
q(\mathcal{I})=\frac{C_{1} e^{M \mathcal{I}}}{\rho^{Q M-M}} \mathcal{I}^{Q-2},
$$

where $\phi_{i}=\ln \psi_{i}$. Recall that the outage probability can be reduced as

$$
\begin{aligned}
& P(\mathcal{I}<Q R / \log e)=\int_{0}^{Q R / \log e} q(\mathcal{I}) d \mathcal{I} \\
= & \frac{C_{1}}{\rho^{Q M-M}}\left(\frac{(Q-2) !}{(-M))^{Q-2}}-e^{\frac{M Q R}{\log e}} \sum_{k=0}^{Q-2} \frac{(Q R / \log e)^{k}}{(-M)^{Q-1-k}}\right) .
\end{aligned}
$$

Substituting $R=r \log \rho$, it can be shown that

$$
P(\mathcal{I}<Q R / \log e) \leq \frac{\rho^{Q M r}}{\rho^{Q M-M}} .
$$

Now the outage probability for the event $\mathcal{A}$ can be written as

$$
\begin{aligned}
P\left(\mathcal{I}_{\mathcal{A}}<Q R\right) & =P(\mathcal{I}<Q R / \log e) P\left\{\left[1+\rho\left|h_{M}\right|^{2}\right]<2^{R}\right\} \\
& \leq \frac{\rho^{(Q+1) M r}}{\rho^{Q M}} .
\end{aligned}
$$

Comparing the outage probability for the case $\mathcal{A}=\{i, i \neq M\}$, the outage probability for the case $\mathcal{A}=\{M\}$ is the dominant one and the lemma is proved.

Proof of Lemma 5: Here we use mathematical induction to prove this lemma. We treat the determinant $\mathcal{I}_{P}=\operatorname{det}\left\{\mathbf{I}_{P}+\right.$ $\left.\rho \Theta_{P} \Theta_{P}^{H}\right\}$ as a function of $P$.

1) For $P=1$, the matrix $\Theta_{P}$ becomes a column vector, $\boldsymbol{\Theta}_{P}=\left[h_{1} g_{1}\right]^{T}$. So the determinant can be written as 
$\mathcal{I}_{P}=\rho\left|h_{1}\right|^{2}+\left(1+\rho\left|g_{1}\right|^{2}\right)$. Hence for $P=1$, the lemma holds.

2) Assume that for $P=l$, the following inequality holds

$$
\mathcal{I}_{l} \geq \prod_{i=1}^{l} \rho\left|h_{i}\right|^{2}+\prod_{i=1}^{l}\left(1+\rho\left|g_{i}\right|^{2}\right) \text {. }
$$

The aim is to show such an inequality also holds for $P=l+1$ given the fact provided in (27). First rewrite the matrix $\Theta_{l}$ as

$$
\boldsymbol{\Theta}_{l}=\left[\begin{array}{cccc}
h_{1} & 0 & 0 & 0 \\
g_{1} & h_{2} & 0 & 0 \\
\vdots & \ddots & \ddots & \vdots \\
0 & \cdots & g_{l-1} & h_{l} \\
0 & \cdots & 0 & g_{l}
\end{array}\right]
$$

When $P=l+1$, the new matrix $\Theta_{l+1}$ can be written as

$$
\boldsymbol{\Theta}_{l+1}=\left[\begin{array}{cc}
\boldsymbol{\Theta}_{l} & \mathbf{h}_{l+1} \\
\mathbf{0}_{l} & g_{l+1}
\end{array}\right]
$$

where $\mathbf{0}_{l}$ is $1 \times l$ all-zero vector and $\mathbf{h}_{l+1}=\left[\mathbf{0}_{l} h_{l+1}\right]^{T}$. As a result, the determinant for $P=l+1$ becomes

$$
\begin{aligned}
\mathcal{I}_{l+1}= & \log \operatorname{det}\left(\mathbf{I}_{l+1}+\rho \boldsymbol{\Theta}_{l+1}^{H} \boldsymbol{\Theta}_{l+1}\right) \\
= & \log \left[\operatorname { d e t } ( \mathbf { I } _ { l } + \rho \boldsymbol { \Theta } _ { l } ^ { H } \boldsymbol { \Theta } _ { l } ) \left(1+\rho\left(\left|h_{l+1}\right|^{2}\right.\right.\right. \\
& \left.\left.+\left|g_{l+1}\right|^{2}\right)-\rho^{2}\left|g_{l}\right|^{2}\left|h_{l+1}\right|^{2}\right]
\end{aligned}
$$

Since $\mathcal{I}_{l} \geq \prod_{i=1}^{l} \rho\left|h_{i}\right|^{2}+\prod_{i=1}^{l}\left(1+\rho\left|g_{i}\right|^{2}\right)$, we have

$$
\mathcal{I}_{l+1} \geq \prod_{i=1}^{l+1} \rho\left|h_{i}\right|^{2}+\prod_{i=1}^{l+1}\left(1+\rho\left|g_{i}\right|^{2}\right) \text {. }
$$

Summarizing these two steps, the inequality derived in Lemma 5 holds for all integer $P$.

Proof of Lemma 6: Recall that the user set $\mathcal{A}$ associated with $\mathcal{O}_{3}$ is characterized by its dimension, $1<|\mathcal{A}|<M$. Without loss of generality, we assume that the set $\mathcal{A}$ consists of several disjoint smaller sets $\mathcal{A}=\left\{\mathcal{A}_{1}, \ldots, \mathcal{A}_{I}\right\}$, where each small user set contains several continuously indexed users. For example, consider $M=7$ and the user set $\mathcal{A}=\{1,2,4,5,7\}$ can be grouped into three smaller sets $\mathcal{A}_{1}=\{1,2\}, \mathcal{A}_{2}=$ $\{4,5\}$ and $\mathcal{A}_{3}=\{7\}$.

According to whether a small set contains the $M$-th user, we can have the following two categories.

1) The small sets do not contain the $M$-th user: Without loss of generality, consider a small user set contains the continuously indexed users from $n$ to $l, \mathcal{A}_{i}=\{n, \ldots, l\}$. Consider the following mutual information based on this user set

$$
\mathcal{I}_{i}=\log \operatorname{det}\left\{\mathbf{I}_{Q(l-n)}+\mathbf{H}_{i}^{H} \mathbf{H}_{i}\right\}
$$

where the channel matrix is defined as $\mathbf{H}_{i}=$ $\operatorname{diag}\left\{\tilde{\mathbf{H}}_{Q}, \ldots, \tilde{\mathbf{H}}_{1}\right\}$ and

$$
\tilde{\mathbf{H}}_{j}=\left[\begin{array}{cccc}
h_{n} & 0 & 0 & 0 \\
g_{j M-n} & h_{n+1} & 0 & 0 \\
\vdots & \ddots & \ddots & \vdots \\
0 & \cdots & g_{j M-l+1} & h_{l} \\
0 & \cdots & 0 & g_{j M-l}
\end{array}\right] .
$$

Note that the structure of $\tilde{\mathbf{H}}_{i}$ fits the requirement of Lemma 5 . Hence by applying this lemma, we can obtain an upper bound for $\mathcal{I}_{i}$ as

$\mathcal{I}_{i} \geq \log \left\{\left(\prod_{i=n}^{l} \rho\left|h_{i}\right|^{2}\right)^{Q}+\prod_{j=1}^{Q}\left(\prod_{i=j M-n}^{j M-l}\left(1+\rho\left|g_{i}\right|^{2}\right)\right)\right\}$.

Using such an inequality, the outage probability associated with this small set $\mathcal{A}_{i}$ can be upper bounded as

$$
\begin{aligned}
P\left(\mathcal{I}_{i}<\right. & (l-n) Q R) \leq P\left\{\prod_{i=n}^{l} \rho\left|h_{i}\right|^{2}<2^{(l-n) R}\right\} \\
& \times P\left\{\prod_{j=1}^{Q}\left[1+\rho\left|g_{j M-l}\right|^{2}\right)<2^{(l-n) Q R}\right\},
\end{aligned}
$$

which has a similar form to the probability of the outage event $\mathcal{O}_{1}$. Hence applying the steps similar to the ones in the proof for Lemma 3, the two probabilities in the above equation can be bounded as

$$
P\left\{\prod_{i=n}^{l} \rho\left|h_{i}\right|^{2}<2^{(l-n) R}\right\} \doteq \frac{\rho^{|\mathcal{S}| r}}{\rho^{l}},
$$

and

$$
P\left\{\prod_{j=1}^{Q}\left[1+\rho\left|g_{j M-l}\right|^{2}\right)<2^{(l-n) Q R}\right\} \doteq \frac{\rho^{Q M r}}{\rho^{Q M-l}} .
$$

And hence the outage probability for a small user subset $\mathcal{A}_{i}$ can be eventually bounded as

$$
P\left(\mathcal{I}_{i}<2^{(l-n) Q R}\right) \leq \frac{1}{\rho^{Q M-(Q M+M) r}} .
$$

2) A small set contains the $M$-th user: When a small set contains the $M$-th user, its associated channel matrix is different to the case discussed previously, which means that its outage probability has to be studied separately. Without loss of generality, consider a small set contains $(M-n+1)$ continuously indexed users, $\mathcal{A}_{j}=\{n, \ldots, M\}$. Then we can have the following mutual information based this user set

$$
\overline{\mathcal{I}}_{j}=\log \operatorname{det}\left\{\mathbf{I}_{Q(M-n+1)}+\overline{\mathbf{H}}_{j}^{H} \overline{\mathbf{H}}_{j}\right\}
$$

where $\overline{\mathbf{H}}_{j}=\operatorname{diag}\left\{\overline{\mathbf{H}}_{j, Q}, \ldots, \overline{\mathbf{H}}_{j, 1}\right\}$ and

$$
\overline{\mathbf{H}} j, i=\left[\begin{array}{cccc}
h_{n} & 0 & 0 & 0 \\
g_{(i-1) M+n} & h_{2} & 0 & 0 \\
\vdots & \ddots & \ddots & \vdots \\
0 & 0 & g_{(i-1) M+1} & h_{M}
\end{array}\right] .
$$

The sub-matrices of $\overline{\mathbf{H}}_{j, i}$ are still tridiagonal ones. Again applying the property of the tridiagonal matrix,

$\overline{\mathcal{I}}_{j} \geq \prod_{i=n}^{M}\left(1+\rho\left|h_{i}\right|^{2}\right)^{Q}+\left(\prod_{i=1}^{M-n} \rho\left|g_{i}\right|^{2}\right) \prod_{j=2}^{Q}\left(\prod_{i=j M-n}^{j M-l} \rho\left|g_{i}\right|^{2}\right)$

Note that the number of the relay-destination channels is only $(M-n) Q-1$. The outage probability associated with this 
event is

$$
\begin{aligned}
P\left(\mathcal{I}_{j}<\right. & \left.\left|\mathcal{A}_{j}\right| Q R\right) \leq P\left\{\prod_{i=n}^{M} \rho\left|h_{i}\right|^{2}<2^{|\mathcal{S}| R}\right\} \\
& \times P\left\{\prod_{j=2}^{Q}\left[1+\rho\left|g_{j M-M}\right|^{2}<2^{Q R}\right\} .\right.
\end{aligned}
$$

Following the previous discussion, it is straightforward to show

$$
P\left\{\prod_{i=n}^{M} \rho\left|h_{i}\right|^{2}<2^{|\mathcal{S}| R}\right\} \doteq \frac{\rho^{|\mathcal{S}| r}}{\rho^{M}}
$$

and

$$
P\left\{\prod_{j=1}^{Q-1}\left[1+\rho\left|g_{j M}\right|^{2}\right)<2^{|\mathcal{S}| Q R}\right\} \doteq \frac{\rho^{Q M r}}{\rho^{Q M-M}}
$$

Finally the outage probability associated with this particular event $\mathcal{A}_{j}$ can be bounded as

$P\left(\mathcal{I}_{j}<|\mathcal{S}| Q R\right) \doteq \frac{1}{\rho^{Q M-(Q M+|\mathcal{S}|)}} \leq \frac{1}{\rho^{Q M-(Q M+M) r}}$.

Note that such a result is the same as the probability for an outage event associated with the user set with $\mathcal{A}_{i}$.

Recall that the overall user set $\mathcal{A}$ consists of several disjoint smaller sets $\mathcal{A}=\left\{\mathcal{A}_{1}, \ldots, \mathcal{A}_{I}\right\}$. Summarizing the results discussed in the previous two sections, we can find that

$$
P\left(\mathcal{I}_{j}<\left|\mathcal{A}_{j}\right| Q R\right) \doteq P\left(\mathcal{I}_{i}<\left|\mathcal{A}_{i}\right| Q R\right)
$$

for all $i, j \in\{1, \ldots, I\}$. Define the outage event $\mathcal{O}_{\mathcal{A}_{i}}=\left\{\mathcal{I}_{i}<\right.$ $\left.\left|\mathcal{A}_{i}\right| Q R\right\}$. Since the overall outage event is defined as $\mathcal{O}_{3}=$ $\left\{\sum_{i=1}^{I} \mathcal{I}_{j}<\sum_{i=1}^{I}\left|\mathcal{A}_{j}\right| Q R\right\}$,

$$
\mathcal{O}_{3} \subseteq \bigcup_{i=1}^{I} \mathcal{O}_{\mathcal{A}_{i}}
$$

which means that the overall outage probability can be bounded as

$$
\begin{aligned}
P\left(\sum_{i=1}^{I} \mathcal{I}_{j}<\sum_{i=1}^{I}\left|\mathcal{A}_{j}\right| Q R\right) & \leq \sum_{i=1}^{I} P\left(\mathcal{I}_{i}<\left|\mathcal{A}_{i}\right| Q R(30)\right. \\
& \doteq \frac{1}{\rho^{Q M-(Q M+M) r}} .
\end{aligned}
$$

And the lemma is proved.

\section{REFERENCES}

[1] A. Sendonaris, E. Erkip, and B. Aazhang, "User cooperation diversitypart I: system description," IEEE Trans. communications, vol. 51, pp. 1927-1938, Nov. 2003.

[2] J. N. Laneman, D. N. C. Tse, and G. W. Wornell, "Cooperative diversity in wireless networks: Efficient protocols and outage behavior," IEEE Trans. Information Theory, vol. 50, pp. 3062-3080, Dec. 2004.

[3] R. U. Nabar, H. Bolcskei, and F. W. Kneubuhler, "Fading relay channels : performance limits and space-time signal design," IEEE Journal on Select. Areas in Comm., vol. 22, pp. 1099-1109, Aug. 2004.

[4] K. Azarian, H. E. Gamal, and P. Schniter, "On the achievable diversitymultiplexing tradeoff in half-duplex cooperative channels," IEEE Trans. Information Theory, vol. 51, pp. 4152-4172, Dec. 2005.

[5] Y. Liang and G. Kramer, "Rate regions for relay broadcast channels," IEEE Trans. Information Theory, vol. 50, pp. 3517-3535, Feb. 2007.
[6] E. Beres and R. Adve, "Selection cooperation in multi-source cooperative networks," IEEE Trans. Wireless Commnications, vol. 7, pp. 118C127, Jan. 2008

[7] T. Cover and J. Thomas, Elements of Information Theory, 6th ed. Wiley and Sons, New York, 1991.

[8] D. N. C. Tse, P. Viswanath, and L. Zheng, "Diversity-multiplexing tradeoff in multiple-access channels," IEEE Trans. Information Theory, vol. 50, pp. 1859-1874, Sept. 2004.

[9] Z. Ding, T. Ratnarajah, and C. Cowan, "On the diversity-multiplexing tradeoff for wireless cooperative multiple access systems," IEEE Trans. Signal Processing, pp. 4627-4638, Sept. 2007.

[10] Y. Chen, S. Kishore, and J. Li, "Wireless diversity through network coding," in Proceeding of IEEE Wireless Communications and Networking Conference (WCNC), Mar. 2006, pp. 1681 - 1686.

[11] Z. Ding, K. K. Leung, D. L. Goeckel, and D. Towsley, "On the study of network coding with diversity," IEEE Trans. on Wireless Communications, vol. 8, pp. 1247 - 1259, Mar. 2009.

[12] M. Yuksel and E. Erkip, "Multi-antenna cooperative wireless systems: A diversity multiplexing tradeoff perspective," IEEE Trans. Information Theory, Special Issue on Models, Theory, and Codes for Relaying and Cooperation in Communication Networks, vol. 53, pp. 3371-3393, Oct. 2007.

[13] D. Chen, K. Azarian, and J. N. Laneman, "A case for amplifyforward relaying in the block-fading multiaccess channel," IEEE Trans. Information Theory, vol. 54, pp. 3728-3733, Aug. 2008.

[14] H. A. David and H. N. Nagaraja, Order Statistics. John Wiley,Third Edition, 2003.

[15] A. Bletsas, A. Khisti, D. P. Reed, and A. Lippman, "A simple cooperative diversity method based on network path selection," IEEE Journal on Select. Areas in Comm., vol. 24, pp. 659-672, Mar. 2006.

[16] Y. Gong, Z. Ding, T. Ratnarajah, and C. Cowan, "Novel channel estimator for a superposition-based cooperative system," in Proc. European Signal Processing Conference (EUSIPCO-06), Sept. 2006, pp. 169-172.

[17] L. Zheng and D. N. C. Tse, "Diversity and multiplexing : a fundamental tradeoff in multiple antenna channels," IEEE Trans. Information Theory, vol. 49, pp. 1073-1096, May 2003.

[18] Z. Ding, Y. Gong, T. Ratnarajah, and C. Cowan, "On the performance of opportunistic cooperative wireless networks," IEEE Trans. on Communications, vol. 56, pp. 1236-1240, Aug. 2008.

[19] G. H. Golub and C. F. van Loan, Matrix computations, 3rd ed. John Hopkins Univerity press, 1996.

[20] S. Yang and J.-C. Belfiore, "Towards the optimal amplify-and-forward cooperative diversity scheme," IEEE Trans. Information Theory, vol. 53, pp. 3114-3126, Sept. 2007

[21] T. Ratnarajah and R. Vaillancourt, "Quadratic forms on complex random matrices and multiple antenna systems," IEEE Trans. Information Theory, vol. 51, pp. 2976-2984, Aug. 2005.

[22] I. S. Gradshteyn and I. M. Ryzhik, Table of Integrals, Series and Products, 6th ed. Academic Press, 2000.

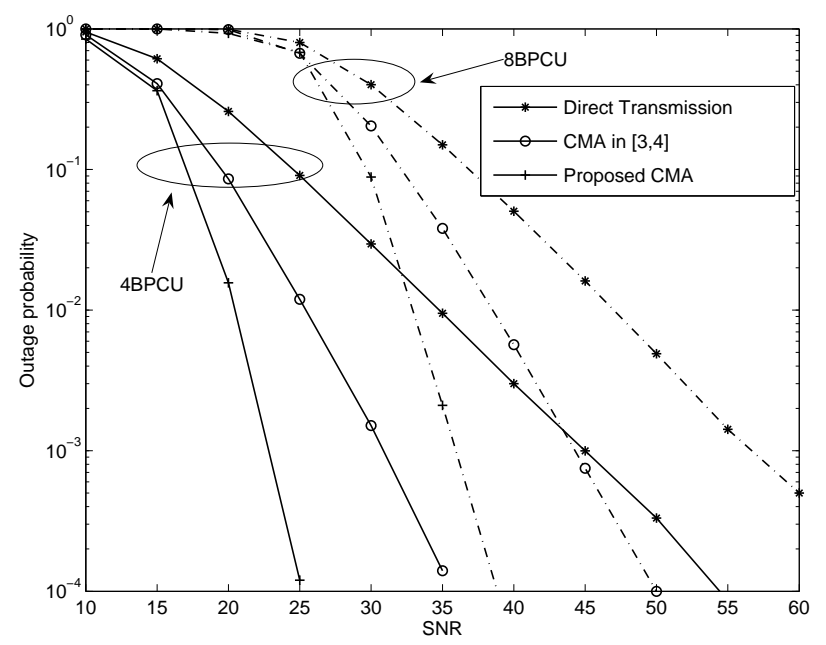

Fig. 1. The outage probability for the proposed scheme, the superposition cooperative scheme [4], [9] and a non cooperative scheme. 


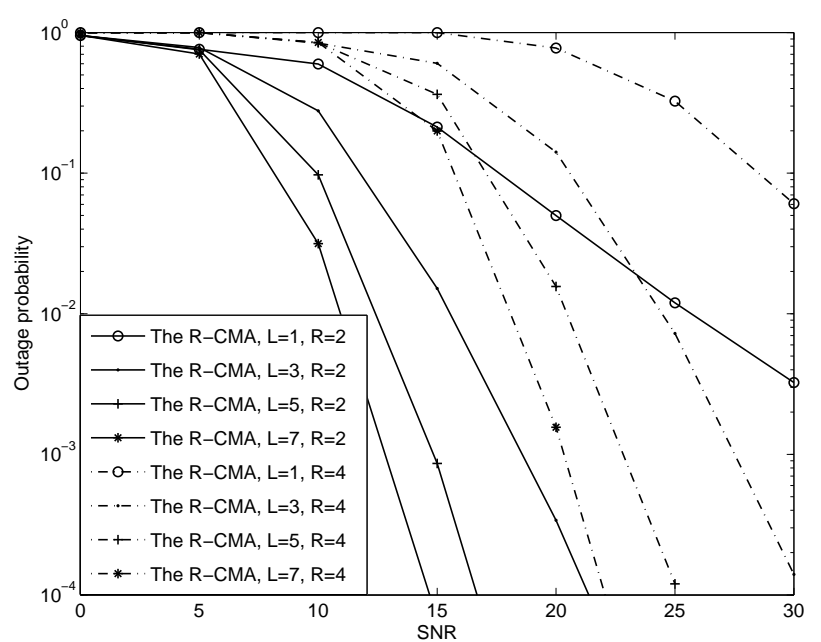

Fig. 2. The outage probability for the proposed CMA scheme with different choices of the relay numbers $L$.

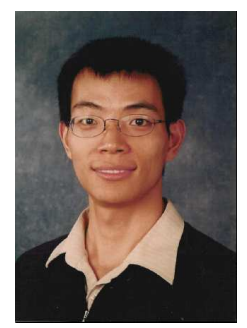

Zhiguo Ding (S'03-M'05) received his B.Eng in Electrical Engineering from the Beijing University of Posts and Telecommunications in 2000, and the $\mathrm{Ph} . \mathrm{D}$ degree in Electrical Engineering from Imperial College London in 2005.

From 2000 to 2002, he was a soft engineer in Datang Telecom Inc. and Vimicro Corporation, Beijing, P.R.China. From Jul. 2005 to Sept. 2007, he was with Queen's University Belfast and Imperial College as a postdoctoral researcher. Since Oct. 2008, he has been with the Department of Communication Systems, Lancaster University, as a Lecturer. His research interests are cross-layer optimization, cooperative diversity, statistical signal processing and information theory.

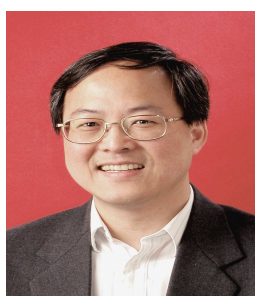

Kin K. Leung received his B.S. degree (with firstclass honors) from the Chinese University of Hong Kong in 1980, and his M.S. and Ph.D. degrees in computer science from University of California, Los Angeles, in 1982 and 1985, respectively.

He started his career at AT\&T Bell Labs in 1986 and worked at its successor companies, AT\&T Labs and Bell Labs of Lucent Technologies, until 2004. Since then, he has been the Tanaka Chair Professor in Internet Technology at Imperial College in London. His research interests include network resource allocation, MAC protocol, TCP/IP protocol, distributed optimization algorithms, mobility management, network architecture, real-time applications and teletraffic issues for broadband wireless networks, wireless sensor and adhoc networks. He is also interested in a wide variety of wireless technologies, including IEEE 802.11, 802.16, and 3G and future generation cellular networks.

He received the Distinguished Member of Technical Staff Award from AT\&T Bell Labs in 1994, and was a co-recipient of the 1997 Lanchester Prize Honorable Mention Award. He was elected in 2001 as an IEEE Fellow for his contributions to performance analysis, protocol design, and control algorithms for communications networks. He receives the Royal Society Wolfson Research Merits Award from 2004 to 2009. He has published widely and acquired patents in many areas of communication networks. He has actively served on many conference committees, including as the committee co-chair for the Multiaccess, Mobility and Teletraffic for Wireless Communications (MMT'98), the Wireless Networking Theory Symposium of IEEE ICC 2002 and the First Annual Conference of International Technology Alliance (ITA) 2007 (sponsored by the U.S. Army and U.K. Ministry of Defence). He was a guest editor for the IEEE Journal on Selected Areas in Communications (JSAC), IEEE Wireless Communications and the MONET journal, and as an editor for the JSAC: Wireless Series. Currently, he is an editor for the IEEE Transactions on Communications, the Transactions on Wireless Communications and International Journal on Sensor Networks.

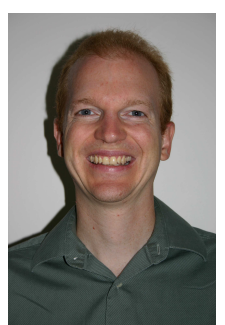

Dennis Goeckel split time between Purdue University and Sundstrand Corporation from 1987-1992, receiving his BSEE from Purdue in 1992. From 1992-1996, he was a National Science Foundation Graduate Fellow and then Rackham Pre-Doctoral Fellow at the University of Michigan, where he received his MSEE in 1993 and his Ph.D. in 1996, both in Electrical Engineering with a specialty in Communication Systems. In September 1996, he joined the Electrical and Computer Engineering department at the University of Massachusetts, where he is currently a Professor. His current research interests are in the areas of communication systems and wireless network theory.

Dr. Goeckel was the recipient of a 1999 CAREER Award from the National Science Foundation for "Coded Modulation for High-Speed Wireless Communications". He was a Lilly Teaching Fellow at UMass-Amherst for the 2000-2001 academic year and received the University of Massachusetts Distinguished Teaching Award in 2007. He served as an Editor for the IEEE Journal on Selected Areas in Communications: Wireless Communication Series during its transition to the IEEE Transactions on Wireless Communications from 1999-2002, and as a Technical Program Committee Co-Chair for the Communication Theory Symposium at IEEE Globecom 2004. He is currently an Editor for the IEEE Transactions on Communications and a CoChair for the Wireless Communications Symposium at IEEE Globecom 2008.

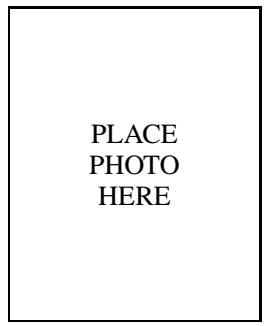

Don Towsley holds a B.A. in Physics (1971) and a Ph.D. in Computer Science (1975) from University of Texas. He is currently a Distinguished Professor at the University of Massachusetts in the Department of Computer Science. He has held visiting positions at IBM T.J. Watson Research Center, Yorktown Heights, NY; Laboratoire MASI, Paris, France; INRIA, Sophia-Antipolis, France; AT\&T Labs - Research, Florham Park, NJ; and Microsoft Research Lab, Cambridge, UK. His research interests include networks and performance evaluation.

$\mathrm{He}$ currently serves as Editor-in-Chief of IEEE/ACM Transactions on Networking, and on the editorial boards of Journal of the ACM and IEEE Journal on Selected Areas in Communications and has previously served on numerous other editorial boards. He was Program Co-chair of the joint ACM SIGMETRICS and PERFORMANCE '92 conference and the Performance 2002 conference. He is a member of ACM and ORSA.

He has received the 2007 IEEE Koji Kobayashi Award, the 2007 ACM SIGMETRICS Achievement Award, the 1998 IEEE Communications Society William Bennett Best Paper Award, and numerous conference/workshop best paper awards. Last, he has been elected Fellow of both the ACM and IEEE. 